\title{
Kinetic Modeling of the Autotrophic Growth of Pavlova lutheri: Study of the Combined Influence of Light and Temperature
}

\author{
Ana P. Carvalho and F. Xavier Malcata* \\ Escola Superior de Biotecnologia, Universidade Católica Portuguesa, Rua Dr. António Bernardino de AImeida, \\ P-4200-072 Porto, Portugal
}

\begin{abstract}
The optimization and control of biochemical processes require the previous establishment of mathematical models that can describe the effect of process variables on their actual kinetics. Environmental temperature is a modulating factor to which the al gal cells respond continuously by adjusting their rates of cellular reactions, their nutritional requirements, and, consequently, their biomass composition. Light intensity is an exhaustible resource, indispensable to autotrophic organisms. The effects of light intensity and temperature on growth of the microalga Pavlova Iutheri, which have hardly been considered to date in a simultaneous fashion, were experimentally assessed using a factorial experimental design; in this way, the effects of each variable independently and their interactions could be quantified, using maximum biomass $\left(X_{\max }\right)$ or maximum specific growth rate $\left(\mu_{\max }\right)$ as objective functions. The preliminary results produced indicated that light intensity plays a more important role on $\mu_{\max }$ than temperature; in the case of $X_{\max }$, both temperature and, to a lesser extent, light intensity do apparently play a role. The highest values of $X_{\max }$ were associated with low temperatures and high light intensities; a similar behavior could be observed for $\mu_{\max }$ concerning light intensity, although the dependency on temperature did not seem to be as important. A more complex mechanistic model was then postulated, incorporating light and temperature as input variables, which was successfully fitted to the experimental data generated during batch cultivation of $\mathrm{P}$. Iutheri.
\end{abstract}

\section{Introduction}

Microalgae are currently employed in such diverse fields as agriculture, wastewater treatment, aquaculture, bioremediation, and manufacture of fine chemicals (1). Among the latter, the Prymnesiophyceae Pavlova I utheri is being studied as a potential source of the polyunsaturated fatty acids eicosapentaenoic acid (EPA) and docosahexaenoic acid (DHA), which are in high demand by the market. These fatty acids bring about health benefits to the human organism, so they are a point of great interest to the food industry, where they act as functional ingredients (2). However, the feasi bility of their industrial manufacture is dependent on their production costs, which can be rationally minimized only if a deeper knowledge of the independent and combined effects of several key parameters upon growth is available.

When a microalgal cell is placed in a given environment that is adequate in terms of prevailing physicochemical conditions, it will grow exponentially until a processing parameter becomes growth-limiting. The uptake of nutrients from the surroundings and the release of cell metabolites thereto occur at rates that depend on internal cell control mechanisms, including adaptability to environmental conditions and genetic heritage. Even

\footnotetext{
* To whom correspondence should be addressed. Email: xmalcata@esb.ucp.pt.
}

if the intrinsic characteristics of the cell population remain somewhat constant, the culture medium can be engineered in terms of several parameters (e.g., temperature, $\mathrm{pH}$, and incident light intensity), whereas certain phenomena (e.g., acid-base equilibrium, ion strength, gas-liquid equilibrium, and rheological properties) eliminate further degrees of freedom (3). This metabolic complexity makes it rather difficult to describe cell kinetics as related to the combined effects of physical and chemical parameters. However, as pointed out before, efficient design and control of a photobioreactor demands performant mathematical models, especially if some degree of prediction is sought. Hence, as a starting point, the most important parameters should be related via an unsegregated and unstructured model; the complexity of the physical situation and the intended application of the kinetic model will dictate its further detail.

Light and temperature are major processing factors that affect overall biomass productivity in photosynthetic al gal systems $(4,5)$. While light is used by cells as energy source, the effects of temperature on cell cultures concern mainly two factors: one relates to the temperature dependence of the structure of cell components (especially proteins and lipids), and the other pertains to the temperature coefficients of reaction rates, which in turn depend on the activation energies of those reactions. As a consequence of these primary effects, 
there will be secondary effects on metabolic regulatory mechanisms, specificity of enzyme reactions, cell permeability, and cell composition (6).

Although there are a number of studies available concerning light intensity and temperature effects independently, extrapolation from studies that hold irradiance constant while varying temperature, or instead hold temperature constant while varying irradiance may be misleading. There is indeed evidence that some species may shift their acclimation strategies in response to combination of those parameters in a different way than if they acted independently (7). Other studies concern simul taneous interactions between those factors, but in such a particular way that hampers its application to other growth conditions, because of the need to determine several biochemical or physiological indicators (8). Furthermore, EPA and DHA are mainly produced by the end of the exponential growth phase, which means that if they are to be produced efficiently, a batch culture system should be employed. However, few studies of the kinetics of batch cultures of this microalga are available, a limitation shared by other species (9).

In this research work, the microalga P. Iutheri was subjected to several combinations of light intensity and temperature, laid out as a star factorial scheme; in this way, both the effects of each variable independently and the interactions among them could be quantified in terms of biomass production, first based on an empirical model. The preliminary relationships thus obtained allowed more complex mechanistic models to be proposed afterward and appropriately tested, including variations in the functional forms of the particular dependencies on temperature and light intensity.

\section{Theoretical Considerations}

To describe the growth behavior of $\mathrm{P}$. lutheri, the logistic model of Weiss and Ollis (10) was used as a starting point. This rather simple model, often applied to batch fermentations, describes growth satisfactorily, both in the exponential and stationary phases. Unlike traditional growth models, which typically relate growth only with cell number, this model provides a correction in the form of an inhibitory term, which becomes more important as the stationary phase is approached, viz.

$$
\frac{\mathrm{d} X(\mathrm{t})}{\mathrm{dt}}=\mu_{\max } \mathrm{X}(\mathrm{t})\left(1-\frac{\mathrm{X}(\mathrm{t})}{\mathrm{X}_{\max }}\right)
$$

where $\mu_{\max }$ is the maximum specific growth rate, $X$ denotes biomass, $t$ denotes time, and $X_{\max }$ represents the maximum biomass the culture can support.

Estimates of the parameters $\mu_{\max }$ and $\mathrm{X}_{\max }$ were obtained, for each experiment, by nonlinear regression to the data using the General RE Gression software package, GREG (11), following integration of eq 1. This program performs nonlinear, multiresponse regression analyses to the data, using finite differences as approximants of the derivatives of the objective function with respect to each parameter; the objective function is minimization of the sum of squares of residuals between model and experimental data.

To establish relationships between the input processing parameters (light intensity and temperature) and the output adjustable parameters ( $\mathrm{X}_{\max }$ and $\left.\mu_{\max }\right)$, two different modeling processes were followed: (i) an empirical determination of functional relationships between parameters, followed by (ii) a mechanistic determination of said relationships. Whereas the former describes the data in a useful mathematical relationship without biological significance, the latter derives from theor etical considerations, hence providing interpretations of the responses observed in terms of the underlying physicochemical mechanisms (12).

The empirical approach was implemented by fitting a second-order polynomial equation to the data, viz.:

$$
z=a+b x+c y+d x^{2}+e y^{2}+f x y
$$

where $\mathrm{x}$ and $\mathrm{y}$ denote absolute temperature and light intensity, respectively, $z$ denotes either $X_{\max }$ or $\mu_{\max }$, and $a-f$ are adjustable parameters. Polynomial probability models are the most common type of empirical models (13), although they sometimes suffer from an intrinsic inability to adequately predict the effect of sufficient factor combinations across the entire interval of study (12).

In terms of the mechanistic approach, several proposals for the functionality of the adjustable parameters were checked, in the general form $\mu_{\max }=\mu_{\max }(\mathrm{L}, \mathrm{T})$ and $\mathrm{X}_{\max }=$ $X_{\max }(L, T)$, always based on the integrated form of the biomass balance depicted in eq 1 . The parameter estimates for those various forms were again obtained with the aid of GREG.

\section{Materials and Methods}

Microalga Source. Pavl ova lutheri (strain SMBA 60) was obtained from Instituto Português de Investigação MARítima (IPIMAR, Portugal).

Cultivation Protocol. Cultures were grown in 250$\mathrm{mL}$ Erlenmeyer flasks. The culture medium was ASW (14) with slight modifications: (i) the medium was enriched with $1 \mathrm{mg} / \mathrm{L}$ thiamin- $\mathrm{HCl}, 2 \mu \mathrm{g} / \mathrm{L}$ biotin, and 1 $\mu \mathrm{g} / \mathrm{L}$ vitamin $B_{12}$ (an essential compound for this microal ga) (15); (ii) silicates and glycylglycine were removed, the former because of redundancy and the latter because of toxicity (16); and (iii) EDTA was increased to $50 \mathrm{mg} / \mathrm{L}$ so as to guarantee improved stability of the medium (data not published), whereas Tris- $\mathrm{HCl}$ was added at $1 \mathrm{~g} / \mathrm{L}$. The $\mathrm{pH}$ was adjusted to 8.0 prior to sterilization. The medium was prepared with deionized water and analytical grade chemicals (Merck, Germany).

A set of 38 Erlenmeyer flasks containing the aforementioned cultures were incubated (for each experiment) in a Gallenkamp orbital incubator (Sanyo, UK), at preset temperature and light regimes, as laid out in Figure 1. Light intensity was measured as PAR (Photosynthetic Active Radiation) with an LI-190SA Quantum Sensor (LI-COR), coupled to an LI-1000 data logger. F or the sake of simplicity, values of light intensity are presented in percentage of incident light; the conversion of such relative values $(X)$ to the corresponding absolute values (conveyed as $\mathrm{PAR}$ ) $(\mathrm{Y}$ ) is directly obtained via the experimentally determined relationship $Y=2.97 \mathrm{X}$. The experiment to be run at $14{ }^{\circ} \mathrm{C}$ and $35 \%$ light intensity was not performed because it was a posteriori found that such a region was uninteresting toward high growth (as desired). Initial cell concentrations were similar in all experiments (ca. $4 \times 10^{5}$ cell $\mathrm{mL}^{-1}$ ).

Assaying Procedure. Sampling al ong the incubation time was performed by random selection and recovery of at least two flasks in each day. Along the experiment, the places left empty by the collected flasks were reoccupied with flasks containing sterilized culture with a similar volume, to maintain the self-shading constant.

Determination of cell number was performed with a Neubauer I mproved bright-line haemocytometer (Supe- 


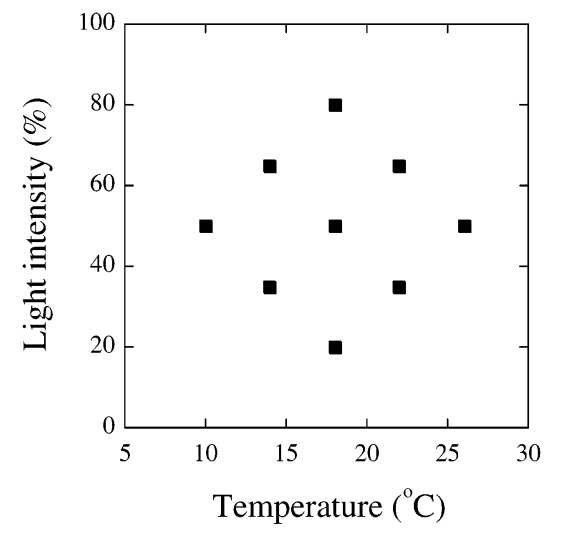

Figure 1. Experimental design followed in the experiments performed at several combinations of light intensity and temperature.

rior, Germany), using appropriate dilutions, under an Olympus $\mathrm{CH}-2$ microscope (J apan).

\section{Results and Discussion}

Monitorization of Biomass Evolution. The variation of biomass during the experiments, expressed in terms of cell number versus time, is depicted in Figure 2. Results were grouped by variation in one single variable (when possible), so as to facilitate inspection and detection of trends. The biomass increased exponentially with time until it reached a plateau, as expected; the time to reach such status depended on the combined conditions of light and temperature set forth. Inspection of the data in Figure 2 indicates that, for a constant light regime of $50 \%$ (Figure $2 a)$, the maximum cell number $\left(X_{\max }\right)$ increases with decreasing temperature; this pattern is also observed for a constant light regime of $65 \%$ (F igure $2 b)$. Regarding the effect of light intensity on $X_{\text {max }}$, at 18 ${ }^{\circ} \mathrm{C}$ the lowest value is obtained at the highest light intensity (80\%) (Figure 2c), but no significant change in performance seems to occur at $22{ }^{\circ} \mathrm{C}$ for the two light intensities tested (35\% and 65\%). This discrepancy in behavior apparently indicates that the effect of light intensity on cell biomass may be temperature-dependent. In attempts to mathematically describe this growth behavior, eq 1 was fitted independently to each dataset (obtained at a given temperature and a given light intensity). The parameter estimates and associated inference intervals are presented in Table 1 . It can be observed that $X_{\max }$ does in fact decrease with increases in temperature, a result consistently noticed at the two light intensities studied. Light intensity has a similar effect, again observed for the two temperatures tested, although less pronounced for the experiments run at 22 ${ }^{\circ} \mathrm{C}$. Regarding $\mu_{\max }$, an inconsistent trend with temperature was observed at $50 \%$ light intensity, but at $65 \%$ there was an increase with temperature. Although in this study one did not measure photosynthetic rates, the observed behavior of maximum specific growth rate with light intensity and temperature resembles trend responses on the photosynthetic rate of Anabaena variabilis with those parameters (6). Since photosynthesis consists of a light reaction (photochemical), which is temperatureindependent, and a dark reaction (chemical), which increases in rate with temperature, changes in temperature may not or may affect the rate of photosynthesis, depending on whether the system is Iight-limited at the time (17). Consequently, the photosynthetic rate (and $\left.\mu_{\text {max }}\right)$ may have not increased with increasing temperature at $50 \%$ light intensity because of the conditions of
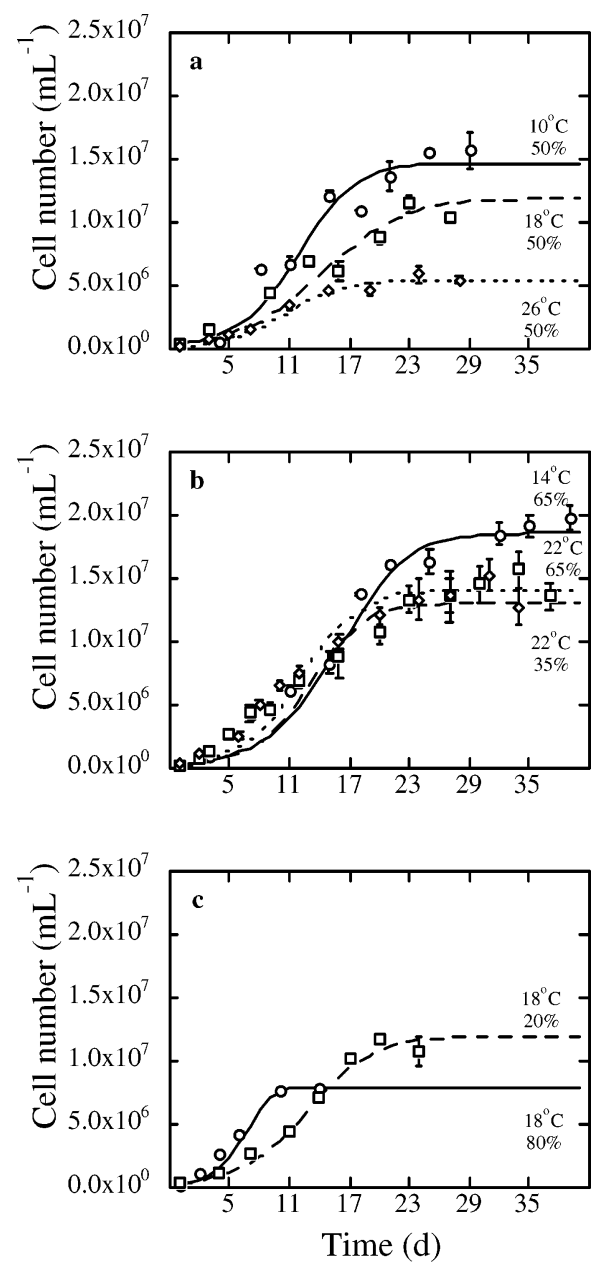

Figure 2. Experimental (symbols) and theoretical (line) cell number concentrations throughout time, using eq 1 at the various combinations of temperature and light intensity tested: (a) experiments run at $50 \%$ light intensity and 10,18 , and 26 ${ }^{\circ} \mathrm{C}$; (b) experiments run at $22{ }^{\circ} \mathrm{C}$, and $35 \%$ or $65 \%$ light intensity, and $14{ }^{\circ} \mathrm{C}$ and $65 \%$ light intensity; and (c) experiments run at $18{ }^{\circ} \mathrm{C}$ and $20 \%$ and $80 \%$ light intensity. Experimental values are presented with $95 \%$ error bars.

Table 1. Parameter Estimates, and Associated 95\% Marginal Inference Intervals (MII) for the Parameters in Eq 1 Fitted to Each Set of Experimental Data

\begin{tabular}{|c|c|c|}
\hline experiment & parametera & estimate \pm MII \\
\hline $10{ }^{\circ} \mathrm{C}, 50 \% \mathrm{~L}$ & $\begin{array}{l}\mu_{\max } \\
\mathrm{X}_{\max }\end{array}$ & $\begin{array}{l}(3.38 \pm 0.49) \times 10^{-1} \\
(1.47 \pm 0.15) \times 10^{7}\end{array}$ \\
\hline $18^{\circ} \mathrm{C}, 50 \% \mathrm{~L}$ & $\begin{array}{l}\mu_{\max } \\
\mathrm{X}_{\max }\end{array}$ & $\begin{array}{l}(2.48 \pm 0.39) \times 10^{-1} \\
(1.19 \pm 0.16) \times 10^{7}\end{array}$ \\
\hline $26^{\circ} \mathrm{C}, 50 \% \mathrm{~L}$ & $\mu_{\max }^{X_{\max }}$ & $\begin{array}{l}(3.36 \pm 0.33) \times 10^{-1} \\
(5.44 \pm 0.42) \times 10^{6}\end{array}$ \\
\hline $14{ }^{\circ} \mathrm{C}, 65 \% \mathrm{~L}$ & $\begin{array}{l}\mu_{\max } \\
\mathrm{X}_{\max }\end{array}$ & $\begin{array}{l}(2.84 \pm 0.33) \times 10^{-1} \\
(1.87 \pm 0.17) \times 10^{7}\end{array}$ \\
\hline $22^{\circ} \mathrm{C}, 65 \% \mathrm{~L}$ & $\begin{array}{l}\mu_{\max } \\
\mathrm{X}_{\max }\end{array}$ & $\begin{array}{l}(3.49 \pm 0.50) \times 10^{-1} \\
(1.31 \pm 0.12) \times 10^{7}\end{array}$ \\
\hline $22{ }^{\circ} \mathrm{C}, 35 \% \mathrm{~L}$ & $\mu_{\max }$ & $\begin{array}{l}(3.35 \pm 0.39) \times 10^{-1} \\
(1.41 \pm 0.11) \times 10^{7}\end{array}$ \\
\hline $18^{\circ} \mathrm{C}, 20 \% \mathrm{~L}$ & $\begin{array}{l}\mu_{\max } \\
\mathrm{X}_{\max }\end{array}$ & $\begin{array}{l}(2.95 \pm 0.23) \times 10^{-1} \\
(1.20 \pm 0.10) \times 10^{7}\end{array}$ \\
\hline $18^{\circ} \mathrm{C}, 80 \% \mathrm{~L}$ & $\begin{array}{l}\mu_{\max } \\
X_{\max }\end{array}$ & $\begin{array}{l}(6.75 \pm 0.58) \times 10^{-1} \\
(7.95 \pm 0.55) \times 10^{6}\end{array}$ \\
\hline
\end{tabular}

low light intensity, but the reverse probably occurred at $65 \%$, as light intensity was then more intense.

Predicted results using the Weiss and Ollis model are also plotted in Figure 2, overlaid on the experimental data for comparison purposes. Since the former overlay 

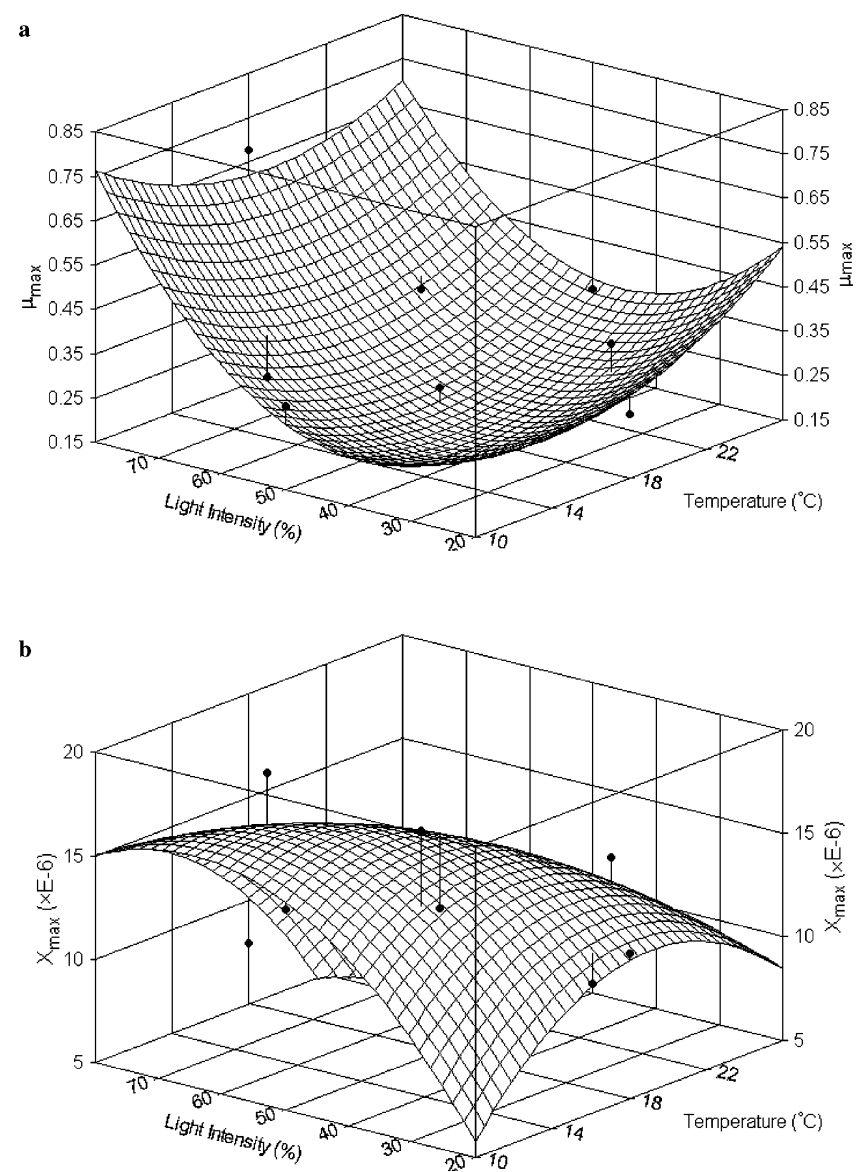

Figure 3. Response surface graphs of the various combinations of temperature and light intensity tested on (a) $\mu_{\max }$ and (b) $X_{\max }$.

quite well on the latter, it can be concluded that the model predictions are satisfactory for all datasets; $X_{\max }$ is particularly well-estimated in all cases, although $\mu_{\max }$ is somewhat underestimated in the experiments performed at $22{ }^{\circ} \mathrm{C}$ and $35 \%$ or $65 \%$ light intensity, as well as in the experiment run at $14{ }^{\circ} \mathrm{C}$.

Determination of Functional Empiric Relationships. To assess the effect of each input variable (i.e., light intensity and temperature) on the model parameters (i.e., $\mathrm{X}_{\max }$ and $\mu_{\max }$ ), the classical one-variable-ata-time study would lead to the "best" values of parameters but only for specific values of the input variables. If those input variables were simultaneously modified, the only possible way to gain knowledge into the nature of the effect of light and temperature would be to reason in terms of a joint functional dependence; such dependence would be better assessed on the basis of experimental data generated by a "star" experimental design. Owing to the existence of three levels of concentration for each variable, the estimated values of $X_{\max }$ and $\mu_{\max }$, as previously obtained with GREG for each set of experimental data, were fitted by the second-order polynomial equation depicted in eq 2 . In this way, the combined influence of light intensity and temperature on the response parameters was duly assessed. The results, expressed in the form of response surface graphs (so as to facilitate observation of the general tendencies of the data), are presented in Figure 3.

On the basis of such plots, a first conclusion can be drawn that relates to the optimum conditions in the light-temperature domain: the highest values of $X_{\max }$ are associated with low temperatures and high light
Table 2. Parameter Estimates, and Associated 95\% Marginal Inference Interval (MII) for the Parameters in Eq 2 Fitted to the Overall Set of Experimental Data ${ }^{a}$

\begin{tabular}{cr}
\hline parameter & \multicolumn{1}{c}{ value \pm MII } \\
a & $\mu_{\max }$ \\
b & $(2.80 \pm 2.02) \times 10^{-1}$ \\
c & $(1.32 \pm 2.00) \times 10^{-1}$ \\
d & $(0.11 \pm 3.16) \times 10^{-1}$ \\
e & $(1.94 \pm 3.16) \times 10^{-1}$ \\
f & $(-0.08 \pm 7.90) \times 10^{-1}$ \\
& $X_{\max }$ \\
a & $(1.43 \pm 1.74) \times 10^{7}$ \\
b & $(-3.85 \pm 13.41) \times 10^{6}$ \\
c & $(-3.43 \pm 134.00) \times 10^{5}$ \\
d & $(-3.33 \pm 24.41) \times 10^{6}$ \\
e & $(-6.44 \pm 53.27) \times 10^{6}$ \\
f &
\end{tabular}

a Note: $x=(T-18) / 8$, with $T$ expressed in ${ }^{\circ} \mathrm{C} ; \mathrm{y}=(\mathrm{L}-50) / 30$, with $\mathrm{L}$ expressed in \%, to normalize the input values; $\mu_{\max }$ is expressed in $\mathrm{d}^{-1} ; \mathrm{x}_{\max }$ is expressed in cell $\mathrm{mL}^{-1}$.

intensities; a similar behavior can be observed for $\mu_{\max }$ concerning light intensity, although the dependency on temperature does not seem to be as important in this case. Surface response trends for light intensity and temperature in terms of effect on the photosynthetic rate of Anabaena variabilis (6) also denoted the dominant influence of light intensity, with optimum values at a combination of high light intensities with low temperatures. Inspection of the parameter values pertaining to $\mu_{\max }$ (see Table 2) demonstrates that both the first- and second-order coefficients associated with temperature (i.e., b and d) are small and are associated with large standard errors. On the other hand, coefficients associated with light intensity (i.e., c and e) exhibit higher values, whereas the term for the interaction thereof (i.e., f) is very low. Hence, given the relatively large variation in growth rate derived from changes in light intensity, any increase in temperature will probably lead to a small increase in biomass productivity; thus, light intensity plays a more important role on $\mu_{\max }$ than does temperature. A somewhat different conclusion can be drawn with regard to $X_{\max }$ : the linear coefficient associated with temperature is larger (about 10-fold) than its light intensity counterpart (see Table 2 ). In the case of $X_{\max }$, both temperature and, to a lesser extent, light intensity apparently do play a role.

At this point, the relative importance of each input parameter upon $\mu_{\max }$ and $X_{\max }$ could be estimated, yet the resulting fit attained was relatively poor, as well as the understanding of the mechanism underlying the observed behavior. Therefore, it was necessary to go one step further and resort to a more mechanistic modeling approach, as detailed below.

Determination of Functional Mechanistic Relationships. (a) Determination of $\mu_{\text {max }}$. Many species of microal gae show similar physiological responses in terms of growth rate to irradiance (for a constant temperature), which are characterized by an essentially proportional relationship (until saturation is reached) (18). On the other hand, the Arrhenius Law (which describes the temperature dependence of chemical reactions in a general fashion) has often been employed by microbiologists in attempts to model bacterial growth as a function of temperature. Such a relationship has the general form

$$
\mu_{\max } \equiv \alpha \cdot \mathrm{e}^{-(\mathrm{E} / \mathrm{RT})}
$$

where $\alpha$ is a preexponential (frequency) factor, E is the 

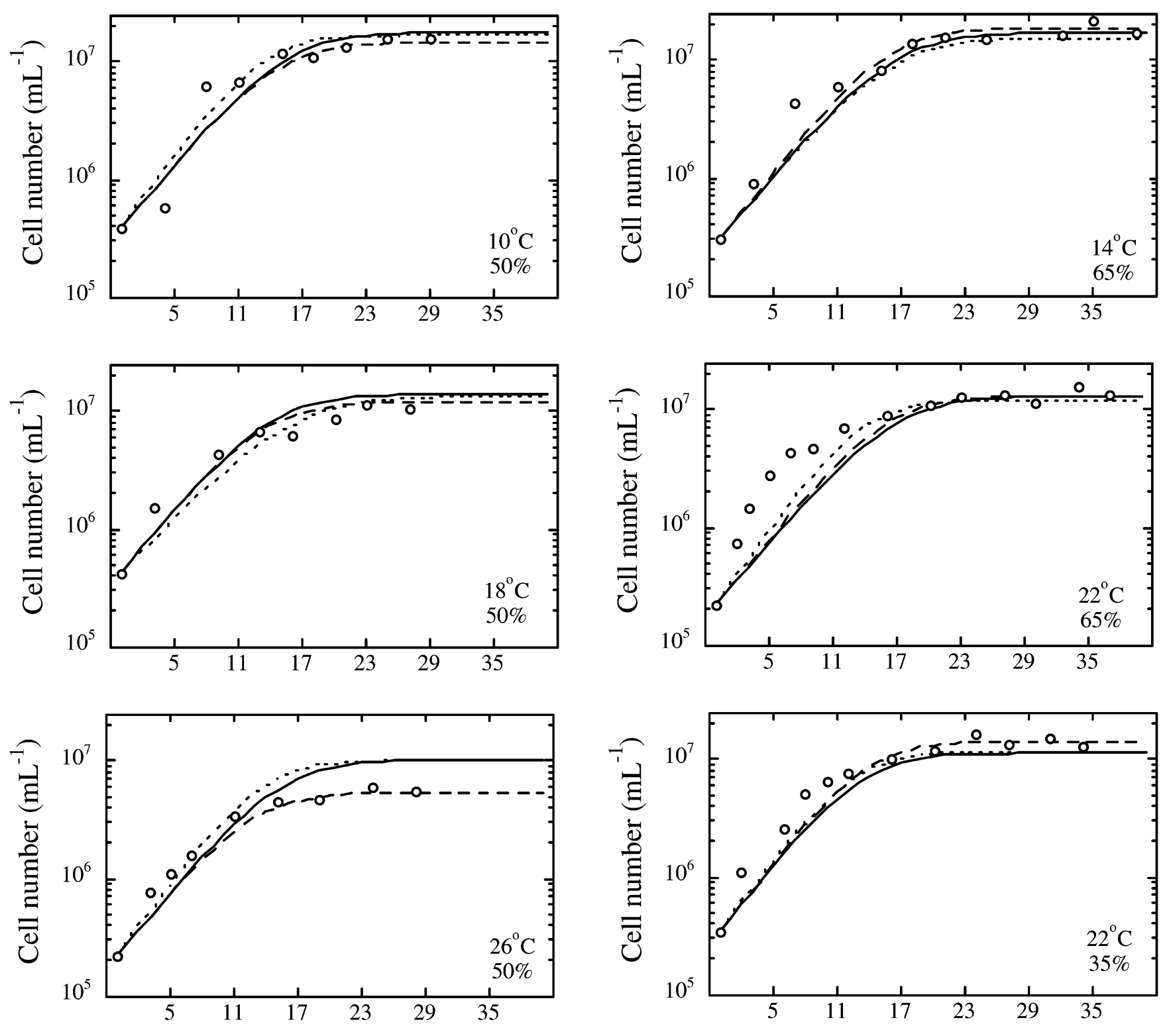

Time (d)

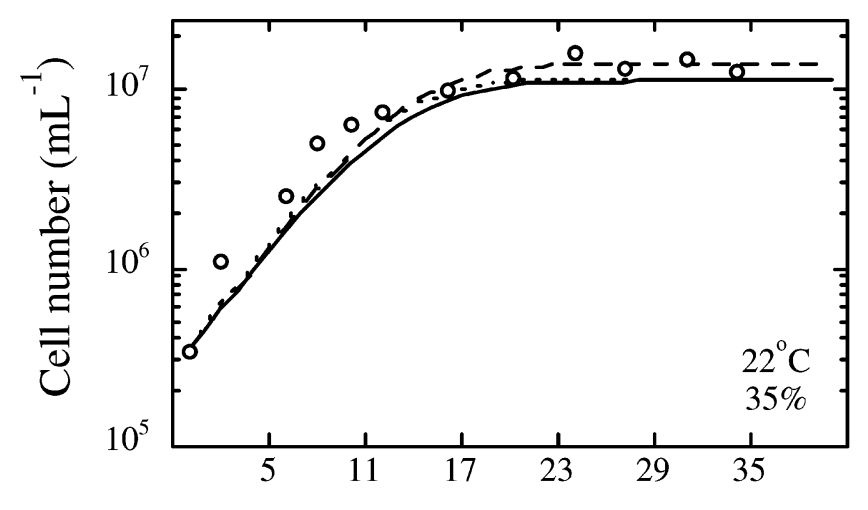

Time (d)
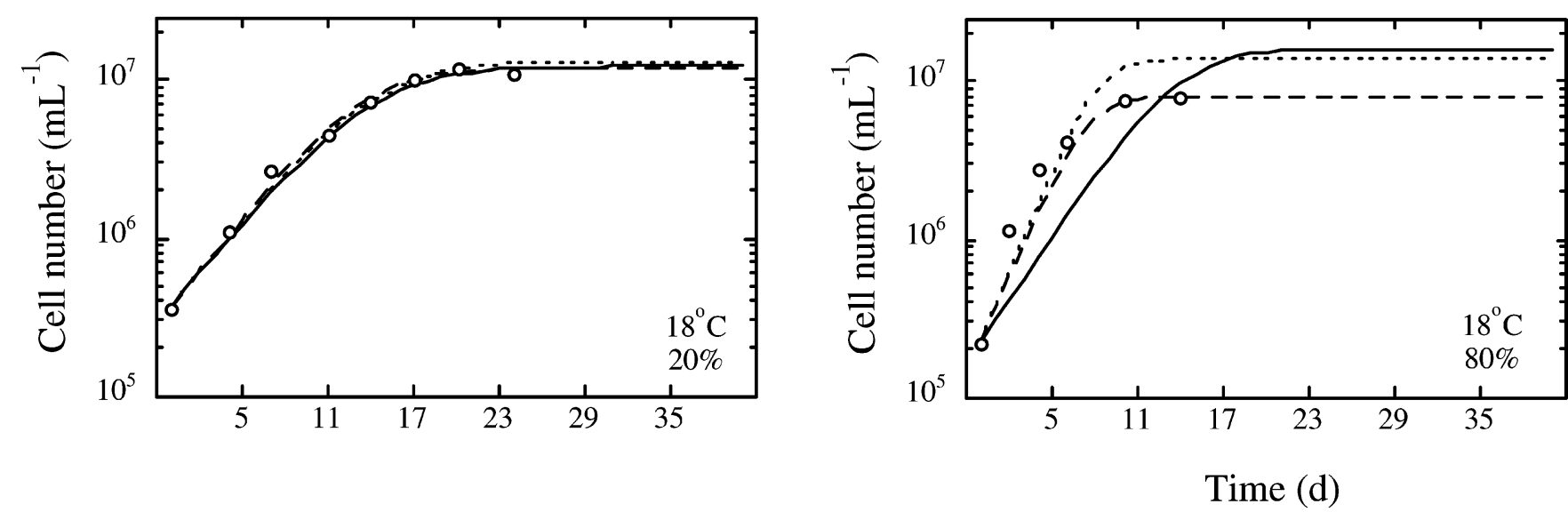

Figure 4. Experimental (symbols) and theoretical (lines) cell number concentrations throughout time, using eqs 1 and 5 ( --- ), eqs 1 and $7(--)$, and eqs 1,5 , and $7(-)$ at the various combinations of temperature and light intensity tested.

activation energy and $\mathrm{R}$ is the ideal gas constant. Neverthel ess, poor fitting is often obtained when trying to apply the Arrhenius Law to experimental data (19). In fact, since the Arrhenius Law is universally valid only for elementary chemical reactions, it is not surprising that such complex biological processes as growth rates will hardly obey such a law (20). Therefore, the limitations of this simplistic form have urged an upgrade of 
Table 3. Functional Forms Postulated for $\alpha(L, T)$ in Eq 4 and Associated Sums of Squares of Residuals ${ }^{a}$

\begin{tabular}{|c|c|}
\hline expression proposed & sum of squares of residuals \\
\hline $\mathrm{K}_{1} \mathrm{~L}$ & $2.53842 \times 10^{3}$ \\
\hline \multicolumn{2}{|l|}{$\overline{\mathrm{T}}$} \\
\hline $\mathrm{K}_{1} \mathrm{~L}$ & $2.53181 \times 10^{3}$ \\
\hline \multicolumn{2}{|l|}{$\overline{\left(K_{2}+L\right) T}$} \\
\hline $\mathrm{K}_{1} \mathrm{~L}$ & $2.51062 \times 10^{3}$ \\
\hline \multicolumn{2}{|l|}{$\overline{\mathrm{K}_{2} \mathrm{~T}+\mathrm{L}}$} \\
\hline $\mathrm{K}_{1} \mathrm{~L}$ & $2.53027 \times 10^{3}$ \\
\hline \multicolumn{2}{|l|}{$\overline{\mathrm{K}_{2}+\mathrm{L}}$} \\
\hline $\mathrm{K}_{1} \mathrm{~L}$ & $2.53735 \times 10^{3}$ \\
\hline$\overline{\mathrm{K}_{2} \mathrm{e}^{-\mathrm{K}_{3} / \mathrm{RT}}+\mathrm{L}}$ & \\
\hline
\end{tabular}

the Arrhenius basic expression. As pointed out previously (21), for a given temperature there seems to be a direct relationship between light intensity and activation energy, which prompts the convenience to consider $\mathrm{E}$ as a light-de-

pendent parameter. On the other hand, the saturation light intensity, consubstantiated in parameter $\alpha$, is also temperature-dependent and should, in general, also depend on light intensity. Hence, eq 3 was conceptually modified in order to incorporate such realizations, viz.

$$
\mu_{\text {max }}(\mathrm{L}, \mathrm{T}) \equiv \alpha(\mathrm{L}, \mathrm{T}) \cdot \mathrm{e}^{-(\mathrm{E}(\mathrm{L}) / \mathrm{RT})}
$$

Several expressions were postulated, departing from eq 4, and duly fitted to the whole experimental dataset, to assess which one would provide the best fit. In a first approach, E was made constant and then set proportional to $L$; $\alpha$ was in both cases set proportional to $L / T$. The sum of squares of the residuals of the model versus the data was lower for the latter, so eq 4 was modified accordingly; various alternative formulations for the functional form of the proportionality constant termed preexponential factor, as depicted in Table 3, were further tested.

From all of the functionalities postulated, the one that yielded the lowest sum of squares of residuals was substituted in eq 4 to give

$$
\mu_{\text {max }}(\mathrm{L}, \mathrm{T}) \equiv \frac{\mathrm{K}_{1} \mathrm{~L}}{\mathrm{~K}_{2} \mathrm{~T}+\mathrm{L}} \cdot \mathrm{e}^{-\left(\mathrm{K}_{3} \mathrm{~L} / \mathrm{RT}\right)}
$$

where $\mathrm{K}_{1}, \mathrm{~K}_{2}$, and $\mathrm{K}_{3}$ are constants; the best estimates of these parameters (and associated 95\% confidence intervals) were $-2.90 \pm 0.50,-0.264 \pm 0.008$, and 141.5 \pm 11.25 , respectively.

The theoretical values predicted using eq 5 are plotted in Figure 4. By visual inspection and comparison with
Figure 2, it can be stated that the differences are indeed minor. To further confirm whether this modified model fits the dataset adequately, a likehood ratio test was used. This test leads to an assessment of the extra sum of squares due to the extra parameters involved in going from the compl ete model, i.e., eq 1, applied independently to eight partial datasets, to the partial model, i.e., eqs 1 and 5, applied simultaneously to the whole dataset. The results of the extra sum of squares analyses involved in going from the complete model (16-parameter model, 2 for each set of 8 experiments) to the partial model (3parameter model for all sets of experiments) are summarized in Table 4. Inspection of this table indicates that the partial model is statistically not different from the full model, so the former suffices to describe the overall dataset from a statistical standpoint.

A model based on a linear relationship of the square root of $\mu_{\max }$ with temperature, viz.

$$
\sqrt{\mu_{\max }}=\alpha+\beta T
$$

where $\alpha$ and $\beta$ are constants and $\mathrm{T}$ is the absolute temperature, was also tested. Such model has been claimed (20) to provide excellent fits to data encompassing bacterial growth, although it possesses no underlying theoretical explanation. The best estimates of $\alpha$ and $\beta$ (and associated 95\% confidence intervals) were $0.107 \pm$ 0.9750 and $(-2.309 \pm 3.367) \times 10^{-3}$, respectively, whereas the sum of squares of the residuals of the model versus the data was $2.55980 \times 10^{3}$. Such overall poor fitting is not surprising, since the model only accounts for the influence of temperature while light intensity is of utmost importance for autotrophic organisms.

The model that yielded the best fit unfolds a Monodtype behavior regarding light intensity. Such finding is somewhat reasonable if one realizes that light is an essential "nutrient" for autotrophic cells, and that light availability (in a batch culture) decreases with time as any other nutrient from the culture medium, although due to a different reason: the increasing cell density, which blocks access of (putatively constant) incident light. As a result, the photosynthetic rate also decreases, with a concomitant decrease of carbon intake. Therefore, light limitation indirectly leads to an inadequate supply of carbon for photosynthesis, so a similarity emerges be tween carbon stress and photoadaptation. The impact of temperature on $\mu_{\max }$ is weaker than that of light intensity. This realization is not totally unexpected and is consistent with trends al ready perceived in the response surface analysis. The influence of temperature on $\mu_{\max }$ in the case of Thalassi osira pseudonana grown at 10 and $18{ }^{\circ} \mathrm{C}$ was also daimed to be minor (18); more specifically, $P$. Iutheri has been pointed out (4) as representative of a group of microorganisms with relatively poor $\mu_{\text {max }}-\mathrm{vs}-\mathrm{T}$ temperature response.

It is interesting to notice that the model proposed for

\begin{tabular}{|c|c|c|c|c|c|}
\hline source & $\begin{array}{l}\text { sum of squares } \\
\text { of residuals }\end{array}$ & $\begin{array}{l}\text { degrees of } \\
\text { freedom }\end{array}$ & $\begin{array}{l}\text { mean } \\
\text { square }\end{array}$ & $\mathrm{F}_{\text {ratio }}$ & $\begin{array}{c}\text { standard } \mathrm{F}_{\text {ratio }} \\
(5 \% \text { significance level })\end{array}$ \\
\hline extra parameters & $0.21355 \times 10^{3}$ & 13 & 16.4269 & 0.436 & 1.893 \\
\hline $8 \times$ eq 1 & $2.29707 \times 10^{3}$ & 61 & 37.6569 & & \\
\hline eqs 1 and 5 & $2.51062 \times 10^{3}$ & 74 & & & \\
\hline extra parameters & $0.32270 \times 10^{3}$ & 13 & 24.8231 & 0.659 & 1.893 \\
\hline $8 \times$ eq 1 & $2.29707 \times 10^{3}$ & 61 & 37.6569 & & \\
\hline eqs 1 and 7 & $2.61977 \times 10^{3}$ & 74 & & & \\
\hline extra parameters & $0.31309 \times 10^{3}$ & 10 & 31.309 & 0.831 & 1.990 \\
\hline $8 \times$ eq 1 & $2.29707 \times 10^{3}$ & 61 & 37.657 & & \\
\hline eqs 1,5 , and 7 & $2.61016 \times 10^{3}$ & 71 & & & \\
\hline
\end{tabular}

Table 4. Incremental Sum of Squares Analyses in Going from Eq 1 to Eqs 1 and 5, from Eq 1 to Eqs 1 and 7, and from Eq 1 to Eqs 1, 5, and 7 
Table 5. Functional Forms Postulated for $X_{\max }(L, T)$ in Eq 1 and Associated Sums of Squares of Residualsa

\begin{tabular}{lc}
\hline expression proposed & sum of squares of residuals \\
\hline$\left(\mathrm{K}_{4}+\mathrm{K}_{5} T\right)\left(\mathrm{K}_{6}+\mathrm{K}_{7} \mathrm{~L}\right)$ & no convergence \\
$\left(\mathrm{K}_{4}+\mathrm{K}_{5} \mathrm{~T}\right) \cdot \mathrm{e}^{-\mathrm{K}_{6} \mathrm{~L} / \mathrm{RT}}$ & $2.62040 \times 10^{3}$ \\
$\left(\mathrm{~K}_{4}+\mathrm{K}_{5} \mathrm{~T}\right)\left(\frac{\mathrm{K}_{6}+\mathrm{L}}{\mathrm{K}_{7} \mathrm{~L}}\right)$ & no convergence \\
$\left(\mathrm{K}_{4}+\mathrm{K}_{5} \mathrm{~T}\right)\left(\frac{\mathrm{K}_{6} \mathrm{~L}}{\mathrm{~K}_{7} T+\mathrm{L}}\right)$ & no convergence \\
a Note: $\mathrm{K}_{4}, \mathrm{~K}_{5}, \mathrm{~K}_{6}$, and $\mathrm{K}_{7}$ are constants.
\end{tabular}

determination of $\mu_{\max }$ as a function of $\mathrm{L}$ and $\mathrm{T}$ (see eqs 1 and 5) is somewhat similar to another model, initially described by Goldman (21), for calculation of the specific growth rate $(\mu)$ : growth was hypothesized to hold a Monod-type dependency on the limiting nutrient (from the culture medium), whereas $\mu_{\max }$ was reported to hold an Arrhenius-type dependency on temperature. Nevertheless, said expressi on was applied only to experiments run at constant light intensity, whereas in our case it was adapted to variable light intensity conditions.

(b) Determination of $\mathbf{X}_{\max }$. A rationale similar to that encompassing $\mu_{\max }$ was followed in the postulation of a functional dependency of the maximum cell number attained on temperature and incident light. The bibliographic information pertaining to this subject is scarce, so simple models were proposed and duly tested, on the basis of trends observed experimentally. The models tested are tabulated in Table 5. Most of them did not converge to estimates of all parameters, so they were promptly discarded. The only exception was

$$
\mathrm{X}_{\max } \equiv\left(\mathrm{K}_{4}+\mathrm{K}_{5} \mathrm{~T}\right) \cdot \mathrm{e}^{-\left(\mathrm{K}_{6} \mathrm{~L} / \mathrm{RT}\right)}
$$

with best parameter estimates of $(1.46 \pm 0.65) \times 10^{8}$, $-(4.57 \pm 2.16) \times 10^{5}$, and $-(3.15 \pm 10.8)$ for $K_{4}, K_{5}$, and $K_{6}$, respectively. This functional form for $X_{\max }$ was incorporated in eq 1 ; the values obtained thereafter are depicted in Figure 4, laid on the experimental data. The goodness of fit is reasonable in general, except for the experiments run under the extreme values of temperature $\left(26{ }^{\circ} \mathrm{C}\right.$ at $50 \%$ light intensity) and light intensity $\left(80 \%\right.$ at $18{ }^{\circ} \mathrm{C}$ ). To assess the goodness of the fit, nested model statistics were again employed; the results thereof are presented in Table 4. Inspection of this table indicates that the combination of eqs 1 and 7 should be selected to describe the experimental data, because the higher sum of squares of residuals obtained is statistically not significant given the decrease in the number of parameters. The apparent discrepancy between statistical similarity and observed dissimilarity relies solely on the intrinsic variability of our data.

Finally, all pieces of information were collected via lumping eqs 1, 5, and 7 into a model bearing 6 parameters, viz., $\mathrm{K}_{1}, \mathrm{~K}_{2}, \mathrm{~K}_{3}, \mathrm{~K}_{4}, \mathrm{~K}_{5}$, and $\mathrm{K}_{6}$; the best estimates of those parameters (and associated $95 \%$ confidence intervals) are $(-2.72 \pm 0.86),(-2.86 \pm 0.20) \times 10^{-1},(1.29$ $\pm 0.22) \times 10^{2},(1.19 \pm 0.59) \times 10^{8},(-3.69 \pm 1.95) \times 10^{5}$, and $(-1.24 \pm 1.40) \times 10^{1}$, respectively. The predicted values obtained with this set of estimates are plotted also in Figure 4. The extreme environmental conditions of temperature and light intensity cause the highest deviations of the predicted values relative to the actual ones. Although the residuals may look large by visual observation, the analysis of the sum of squares associated with such a model makes it not statistically different from the one by Weiss and Ollis (Table 4); such apparent discrepancy derives again from the intrinsic variability of our data.

\section{Conclusions}

The optimization and control of biochemical processes requires postulation of mathematical models that can describe the kinetics of the relevant process variables. Environmental temperature is a nuclear parameter, as it controls the basic rate of all chemical reactions that take place in algal cells. On the other hand, light intensity is a primordial metabolic resource for autotrophic microorganisms. Our research effort generated the development of mechanistic equations that permit prediction of the maximum growth rate and the maximum biomass of $\mathrm{P}$. lutheri, in response to those combined environmental conditions; the resulting estimates yield good fits to actual experimental data.

\section{Acknowledgment}

Mr. Rui Barros is hereby gratefully thanked for his kind help with the software GREG. Financial support (grant BD/2838/93-IF) by PRAXIS XXI (Portugal) for A.P.C. is also acknowledged.

\section{References and Notes}

(1) Becker, E. W. Large-Scale Cultivation. In Microalgae Biotechnology and Microbiology; Becker, E. W., Ed.; Cambridge University Press: Cambridge, UK, 1994; pp 63-171.

(2) Carvalho, A. P.; Malcata, F. X. Polyunsaturated Fatty Acids and Functional Foods. World Ingredients 1996, March/April, 22-26.

(3) Bailey, J . E.; Ollis, D. F. Biochemical Engineering Fundamentals; Bailey, J. E., Ollis, D. F., Eds.; McGraw-Hill: New York, NY, 1986; pp 373-456.

(4) Goldman, J . C. Temperature Effects on Steady-State Growth, Phosphorus Uptake, and the Chemical Composition of a Marine Phytoplankter. Microb. Ecol. 1979, 5, 153-166.

(5) Thompson, P. A.; Guo, M. Effects of Variation in Temperature. I. On the Biochemical Composition of Eight Species of Marine Phytoplankton. J . Phycol. 1992, 28, 481-488.

(6) Richmond, A. Cell Response to Environmental Factors. In CRC Handbook of Microalgal Mass Culture; Richmond, A, Ed.; CRC Press: Boca Raton, FL, 1986; pp 69-99.

(7) Dermoun, D.; Chaumont, D.; Thebault, J .; Dauta, A. Modelling of Growth of Porphyridium cruentum in Connection with Two Interdependent Factors: Light and Temperature. Bioresour. Technol. 1992, 42, 113-117.

(8) Zhang, X. W.; Zhang, Y. M.; Chen, F. Kinetic Models for Phycocyanin Production by High Cell Density Mixotrophic Culture of the Microalga Spirulina platensis. J . Ind. Microbiol. Biotechnol. 1998, 21, 283-288.

(9) Levert, J. M.; J inlan, X. Modeling the Growth Curve for Spirulina (Arthrospira) maxima, a Versatile Microalga for Producing Uniformly Labelled Compounds With Stable Isotopes. J . Appl. Phycol. 2001, 13, 359-367.

(10) Sattur, A. P.; Karanth, N. G. Mathematical Modelling of Production of Microbial Lipids. Bioprocess Eng. 1991, 6, 227234.

(11) Stewart, W. E.; Caracotsios, M.; Sørensen, J. P. GREG Software Package Documentation. University of Wisconsin: Madison, WI.

(12) McMeekin, T. A.; Olley, J .; Ratkowsky, D. A.; Ross, T. Predictive M icrobiology: Towards the I nterface and Beyond. Int. J . Food Microbiol. 2002, 73, 395-407.

(13) Kirsty, A. P.; Salter, M. A.; Ratkowsky, D. A.; Ross, T. Devel opment of Growth Limits (Growth/no Growth Interface) Modeling and its Application to Predictive Food Microbiology. Rec. Res. Devel. Microbiol. 1999, 3, 535-549.

(14) Borowitzka, M. A. Algal Growth Media and Sources of Algal Cultures. In Micro-algal Biotechnology; Borowitzka M. 
A., Borowitzka L. J ., Eds.; Cambridge University Press: Cambridge, 1988; pp 456-465.

(15) Droop, M. R. Cobalamin requirement in Chrysophyceae. Nature 1954, 174, 520.

(16) Carvalho, A. P.; Malcata, F. X. Effect of Culture Media on Production of Polyunsaturated Fatty Acids by Pavlova I utheri. Cryptogamie Algol. 2000, 21, 59-71.

(17) Spotte, S. Light and Algal Growth. In Seawater Aquariums; Spotte, S., Ed.; J ohn Wiley \& Sons: New York, NY, 1979; pp 10-25.

(18) Thompson, P. The Response of Growth and Biochemical Composition to Variations in Daylength, Temperature, and I rradiance in the Marine Diatom Thalassi osira pseudonana (Bacillariophyceae). J . Phycol. 1999, 35, 1215-1223.
(19) Ratkowsky, D. A.; Lowry, R. K.; McM eekin, T. A.; Stokes, A. N.; Chandler, R. E. Model for Bacterial Culture Growth Rate Throughout the Entire Biokinetic Temperature Range. J . Bacteriol. 1983, 154, 1222-1226.

(20) Ratkowsky, D. A.; Olley, J .; McMeekin, T. A.; Ball, A. Relationship Between Temperature and Growth Rate of Bacterial Cultures. J . Bacteriol. 1982, 149, 1-5.

(21) Goldman, J . C.; Carpenter, E. J . A Kinetic Approach to the Effect of Temperature on Algal Growth. Limnol. Oceanogr. 1974, 19, 756-766. 